\title{
Design and Implementation of a Remote Monitoring System for the Elderly Based on Cloud Architecture
}

\author{
Y.Q Zhao \\ College of Information Engineering Qingdao University \\ China
}

\begin{abstract}
A monitoring system of caring the elderly is designed to solve those problems that occur constantly under the serious aging situation. In the system, the ZigBee technology, sensor technology and cloud platform technology are comprehensively used. The system can monitor the elderly's sleep actions during the night and the activities during the day. Their behavior data can be used to predict health events and provide advice for caregivers. It indicates that the system works well and provides a feasible solution for telecare.
\end{abstract}

Keywords-remote monitoring system; cloud platform; sensor; elderly care; telecare

\section{INTRODUCTION}

According to Chinese sixth national census data, the population over 60 years old is more than 177,594,400[1].In this census, there are 40,134,148 households of the elderly. Among them, the number of the single elderly accounts for 45.5 percent and the number of the elderly couple accounts for 55.5 percent[1]. China will fully enter into the aging society [2].It is the traditional thought of China that most of the old man is not willing to live in nursing homes. So, care for the elderly at home has become a serious social problem[3].

The health status of the elderly is closely related to their daily behaviors. In maintaining the general health of the elderly, it can be useful to remotely monitor their sleep actions and daily activities. Health management can be enhanced if these data can be acquired using fully automated procedure. On the other hand, the development of technology, like low cost sensors, computers, and communication systems, has brought this into reality. And enabling technology has the potential to revolutionize health care services for older adults, promote independence, and enhance aging in place[4].

Based on the above condition, this paper proposes a set of monitoring system based on ZigBee technology and cloud platform technology for the elderly. One advantage of this system is that monitoring can be achieved automatically, without placing any sensors on the subjects. This system applied the ZigBee technology as home wireless network. ZigBeeis a wireless communications standard developed by ZigBee Alliance[5]. Open standard based product, potential for interoperability with devices made by different vendors, overthe-air firmware upgrades are the obvious advantages of ZigBee technology. So, another advantage of this system is the low-cost, easy to expand and upgrade. The cloud platform applies SOA(Service Oriented Architecture) technology. It achieves maximum agility, extensibility and interoperability for future extension.

\author{
Z.Q Yu \\ College of Information Engineering Qingdao University \\ China
}

\section{RELATED WORKS}

There are many monitoring and abnormal detection systems which are designed for the elderly. For example, there is one system that detects the location and dangerous actions of the elderly by capturing the RFID and sensors in their phone[6]. In this system, the elderly should bring a cell phone in which has the sensors put in. In another fall detection system, it requires the elderly to wear shoes in which pressure sensors are put. The results of the detection are not sent to the background management system via network and warnings cannot be sent to care givers of the elderly[7]. In another system, medical devices installed in the old people's home connected to house concentrator via ZigBee to collect patients' basic physiological data[8]. However, the elderly may not use these medical devices themselves.

In foreign countries, monitoring systems for eldercare have been investigated previously. Glascok and Kutzik proposed the use of motion sensors to infer activities of daily living[9]. Ogawa et al. also document an early study in which two individual participants are monitored for motion activity, sleep time, and appliance use (through wattmeters) continuously for over a year[10]. The system applied wired network to connect the sensors and computer.

This paper proposes one system which doesn't require the elderly to bring a cell phone or wear special shoes. When the elderly sleep in their bed or watch TV on the couch, the system can detect the information and send them to the management system via wireless network in real-time. A warning will be given to their children when parents encountered some unexpected conditions.

\section{OVERALL ARCHITECTURE OF THE SYSTEM}

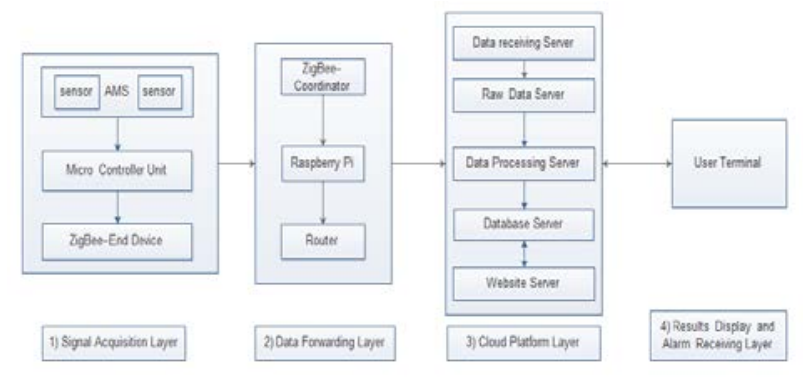

FIGURE I. THE OVERALL ARCHITECTURE

The overall architecture of the proposed monitoring system is depicted in Fig.1. The overall architecture contains four 
layers. They are data acquisition layer, data forwarding layer, cloud platform layer, display of user activity and alarm receiving layer.

Each layer consists of different devices and software. And they communicate in different ways. The first two layer communicate via ZigBee. And the third module communicates with the second layer and the fourth layer via the Internet.

From the picture above, it can be seen that the cloud platform layer is the most complicate and important part of the system. In this paper, it includes five modules. They are data receiving server, database server of raw data, data processing server, database server of processed data and website server. One advantage of the platform is that the web application acquires sensors data by invoking web service interface. It makes application integration much simple.

\section{DeTAILED DESIGN OF THE SYSTEM}

\section{A. Signal acquisition layer}

Auto mat sensor is a film type touch sensor and the touch points are distributing uniformity on the pressed surface of mat. It would send a touch signal when mat get the pressure from exterior. The sensor can be designed footprint and pressure profile according to mat figure, mat rigidity and casing elasticity. In this paper, we put the dynamic pressure sensor under the mattress to monitor the elderly's time of getting up and going to bed. STC12LE5A60S2 MCU reads the changes of the dynamic pressure sensors and sends them in the format of our definition through ZIGBEE END Device which is developed by Shanghai Shun Zhou Network Technology. Addressing space of up to 64 bit IEEE address devices, 65, 535 networks[11]. Each ZigBee END Device has one unique MAC address, we can know where the data come from according to the MAC address.

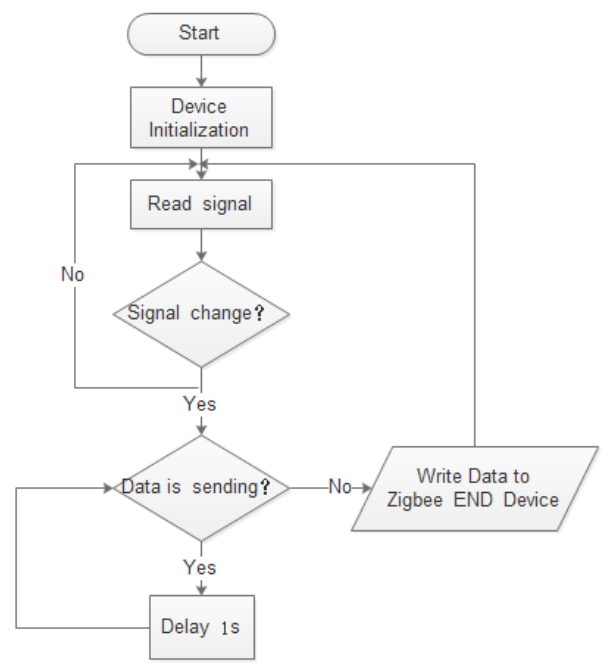

FIGURE II. THE FLOW CHART OF SIGNAL ACQUISITION

We have tested the automatic mat sensor (AMS) under different conditions. We put the AMS in the position of their shoulder and hips when the elderly lie on the bed. And we tested several postures of the two locations. The AMS can monitor different pressure signal in the above conditions and transmit them to data forwarding layer quickly and correctly.

\section{B. Data forwarding layer}

This module is a link between the data acquisition module and the cloud module. It is a very important part of the system. It guarantees correct receiving of data from the AMS and correct transmissions of data to cloud platform.

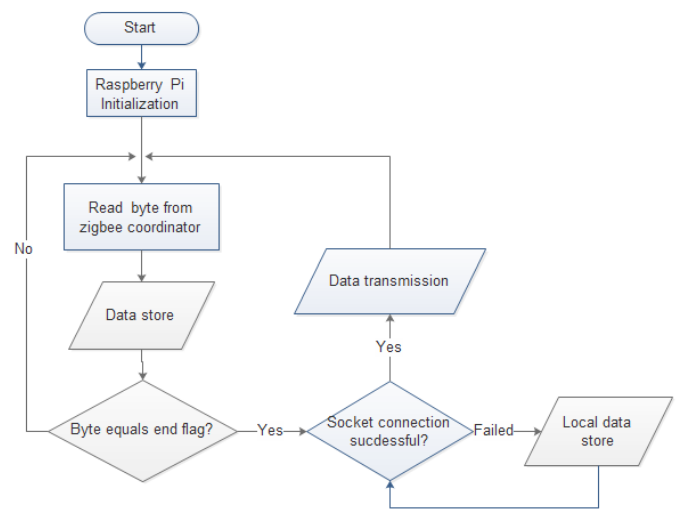

FIGURE III.

THE FLOW CHART OF DATA FORWARDING

In this module, the Raspberry Pifunctions as a forward center of data. It reads data from ZigBee END Device in realtime. Meanwhile it establishes a socket connection to the cloud platform server via the internet. Then the data are packaged into a fixed format and sent to the server if the connection establishes successfully.

\section{Cloud platformlayer}

The cloud platform is short for cloud computing platform. This kind of platform allows developers to run their programs in the "cloud" or use the "cloud" services. Cloud computing architecture mainly can be divided into four levels. They are the presentation layer, the middle tier, the infrastructure layer and the management layer.

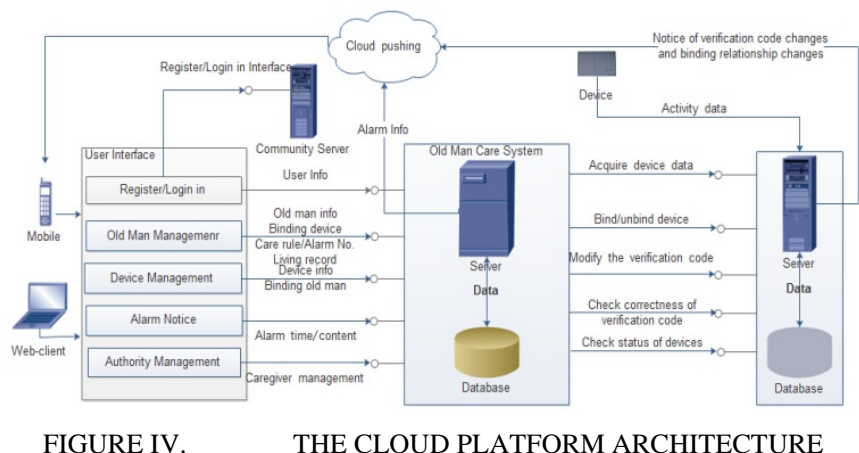

In this paper, it mainly contains above parts. They are raw data server, old man care system platform, and community server. As we can see, the old care man system completes the function of monitoring by invoking services with different interfaces. This method reduces the coupling of different parts and makes the system extension much easier when the system needs adding other functions in the future. 


\section{TEST METHODS AND RESULTS}

The above proposed modules are developed and tested. As the Fig. 2 shows, the test has three different groups. There are six kinds of postures in each group. In the first group, the two pressure mats are parallel. They are at right angles to the testers' body. In the second group, the angle is less than 90 degrees. In the third group, the two mats are on a straight line. The mats and the testers' body form an angle which is less than 90 degrees.
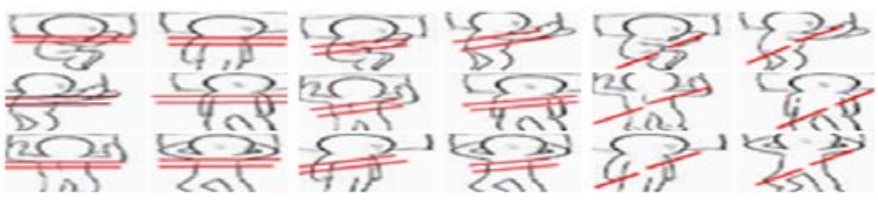

FIGURE V. TEST WAYS

The website is developed to enable the caregivers to check the status of the old man and manage the information of both devices and the old men. The following line chart displays the living routine of one tester on October 1-6, 2014.

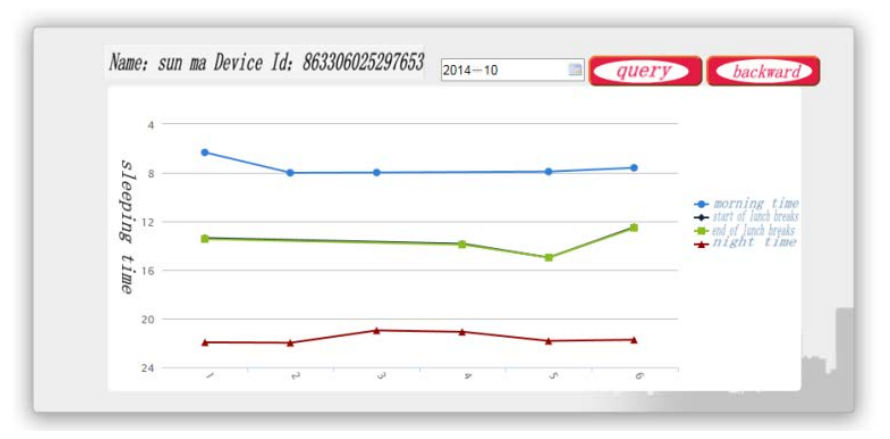

FIGURE VI.

THE ELDERLY SLEEPING TIME

As the chart shows, the four lines represent the getting up time, lunch rest time and the time of going to bed at night. Because the old person sleeps for only a while at noon, the two lines seem to be one line in the line chart. But when we move our mouse on the small square of each day, we can find the start and the end time of the old person's lunch rest. In this chart, we can see that the old person don't go to bed on October 2 and October 3 at noon. It will jump to the detailed page of the day when we click the below number. The detailed page displays the sleep process of the whole day. If the old person haven't got up or went to sleep insetting time, the system will add this content to the notice management part and send a message to the user to remind him of this condition.

\section{CONCLUSIONS}

In this paper, we propose one old person monitoring system. Several testers have been monitoring for over one year. The system obtains the basic information of the old man at home successfully in real-time. Sleeping activities of the testers have been recorded and abnormal behaviors can also be detected automatically. It overcomes the disadvantage of other systems which requiring the old person wearing special devices on their body and realizes telecare of the elderly. The architecture of system enables the future extension easier. In the next stage, we will make the system more perfect by adding more AMS in the old person's home to obtain more information of the old person. We can improve the accuracy of forecast by defining more specific care rules according to more comprehensive behavior information of the elderly.

\section{REFERENCES}

[1] Shasha Peng,Wan Zou. Analysis of the situation and characteristics of population inchina.Industry and Technology Forum.2013

[2] Guangzong Mu,Tuan Zhang.The aging of population in china and its strategy.Journal of Central China Normal University(Humanities and Social Sciences). 2011

[3] Xinai Chang.The elderly care system based on Internet of things. Household Application Trchnology.2011

[4] M.J.Rantz, M. Skubic, S.J. Miller, and J.Krampe. Using Technology to Enhance Aging in Place.

[5] ZigBee Alliance.ZigBee Document 053474r17.ZigBee Alliance Board of Directors.2008

[6] Dong Wang, Wancheng Ge, Yunguang Wang, Weijing Xuan, WenliangGu. Design and implementation of home care system for the elderly. Journal of Clinical Rehabilitative Tissue Engineering Research.2009

[7] Xin Shi, QingyuXiong, Luning Lei. Research on fall detection system based on pressure sensor. Chinese Journal of Scientific Instrument.2010

[8] F.Vergari, V.Auteri, C.Corsi, C.Lamberti. A ZigBee-based ECG transmission for a low cost solution in home care services delivery.Mediterranean Journal of Pacing and Electrophysiology.

[9] Eric Campo, Marie Chan, WalidBourennane, and Daniel Estève.Behaviour monitoring of the elderly by trajectories analysis.32nd Annual International Conference of the IEEE EMBSBuenos Aires, Argentina.2010

[10] Mitsushiro Ogawa, Ryoji Suzuki, SakukoOtake, Takeshi Izutsd, Tsutomu Iwaya, Tatsuo Togawa. Long term remote behavioral monitoring of elderly by using sensors installed in ordinary houses.2nd Annual International IEEE-EMBS Special Topic Conference on Microtechnologies in Medicine \& Biology.2002

[11] Dr.S.S.RiazAhamed.The RoleofZigbee Technologyin Future Data Communication System.Journal of Theoretical and Applied Information Technology. 